\section{Prävention und Behandlung von Thrombosen}

\author{
A. Greinacher ${ }^{1}$ \\ ${ }^{1}$ Institut für Immunologie und Transfusionsmedizin, \\ Ernst-Moritz-Arndt-Universität Greifswald
}

In den 1960er Jahren standen zur Prävention und Behandlung von Thrombosen zwei Medikamentenklassen zur Verfügung, Vitamin-K-Antagonisten und unfraktioniertes Heparin. Beide Klassen sind heute noch Grundpfeiler der antithrombotischen Arzneimitteltherapie, können jedoch erhebliche Nebenwirkungen haben, was eine regelmäßige Überwachung des Patienten erfordert. Meine Forschungsarbeit ist maßgeblich davon geprägt, ein besseres Verständnis dieser unerwünschten Arzneimittelwirkungen zu gewinnen und deren Diagnostik und Behandlung zu verbessern. Die Entwicklung einer Vielzahl neuer gerinnungshemmender Medikamente, vor allem in den letzten 20 Jahren, hat entscheidend zu einer sichereren Behandlung mit gerinnungshemmenden Medikamenten beigetragen. Maßgeblich für die Entwicklung neuer Antikoagulanzien war die Aufklärung der einzelnen Schritte der Gerinnungskaskade, der Struktur des Gerinnungsfaktors $\mathrm{X}$ bzw. Xa in seiner aktivierten Form, sowie des Thrombins. Heute stehen eine Vielzahl von Medikamenten zur Prävention und Behandlung von Thrombosen zur Verfügung, die mindestens so effektiv sind wie Heparin und Vitamin-KAntagonisten, jedoch weniger unerwünschte Wirkungen zeigen und einen geringeren Überwachungsaufwand erfordern. Schritt 1, die niedermolekularen Heparine: Mit der Verfügbarkeit niedermolekularer Heparine hat sich der breite Einsatz der medikamentösen Thromboseprophylaxe bei Krankenhauspatienten durchgesetzt. Ebenso ist die Behandlung von Thrombosen im ambulanten Bereich möglich geworden (da niedermolekulare Heparine auch in therapeutischer Dosierung subkutan appliziert werden, und nicht wie unfraktioniertes Heparin intravenös). Zudem wurde gegenüber dem unfraktionierten Heparin das Risiko schwerer unerwünschter Arzneimittelwirkungen gesenkt: Das Risiko einer Heparin-induzierten Thrombozytopenie wurde um $90 \%$ gesenkt, ebenso wie das Risiko einer Osteoporose bei der längerfristigen Gabe von niedermolekularem Heparin (z. B. zur Prävention und Behandlung von Thrombosen in der Schwangerschaft).

Schritt 2, Biotechnologie und direkte parenterale ThrombinInhibitoren: Nachdem aufgereinigtes Hirudin aus Blutegeln bereits in den 1930er Jahren zur Antikoagulation eingesetzt wurde, stand seit den 1990er Jahren mit rekombinantem Hirudin erstmals ein „neues“ Antikoagulans in ausreichender Menge zur Verfügung. Hirudin hemmt Thrombin sehr effektiv. Allerdings haben Nebenwirkungen wie die Akkumulation bei Niereninsuffizienz, seine Immunogenität und die daraus resultierende schwierige Steuerbarkeit sowie ein fehlendes Antidot dazu geführt, dass rekombinantes Hirudin wieder vom Markt genommen wurde. Hingegen hat das Derivat des Hirudins, Bivalirudin, bei welchem die aktiven Gruppen des Hirudins mit einem Spacer miteinander verbunden sind, vor allem in Nordamerika weiterhin einen festen Stellenwert bei der Behandlung von Patienten mit akutem Koronarsyndrom. Schritt 3, Arzneimitteldesign und direkte orale Antikoagulantien: Die Kenntnis der molekularen Struktur von Thrombin und Faktor Xa ermöglichte die Entwicklung oral verfügbarer direkter Antikoagulanzien. Bereits der erste direkte Thrombininhibitor, Ximelagatran, zeigte eine bessere Wirksamkeit als niedermolekulares Heparin bei der Vermeidung von Thrombosen nach großen orthopädischen Operationen und als Vitamin-K Antagonisten bei der Prävention von Schlaganfällen bei Patienten mit Vorhofflimmern. Ximelagatran musste allerdings aufgrund erhöhter Lebertoxizität vom Markt genommen werden. Auch das zweite direkt wirksame, orale Antikoagulans, Dabigatran, ist ein Thrombininhibitor. Dabigatran wird heute eingesetzt für die Thromboseprophylaxe bei orthopädischen Patienten und für die Antikoagulation bei Patienten mit Vorhofflimmern bzw. Patienten mit Thrombosen oder Lungenembolie. Neben direkten Inhibitoren des Thrombins wurden direkte Inhibitoren des Faktors Xa entwickelt. Rivaroxaban, Apixaban und Edoxaban sind mittlerweile zugelassen, und weitere Substanzen befinden sich in der Entwicklung. Alle direkten oralen Antikoagulanzien werden in einer festen Dosis gegeben und erfordern keine regelmäßige Überprüfung des Medikamentenspiegels. Im Gegensatz zu den Vitamin-K-Antagonisten ist ihre Interaktion mit anderen Medikamenten gering und auf wenige Medikamentengruppen beschränkt. Ihre Interaktion mit Nahrungsmitteln ist vernachlässigbar. Diese Vorteile sind für die Patienten mit einer deutlichen Verbesserung ihrer Lebensqualität verbunden. Allerdings können diese Medikamente bei Patienten mit Nierenfunktionseinschränkungen gefährlich akkumulieren.

Die direkten oralen Antikoagulanzien wurden in methodisch hochwertigen, großen Studien an mehreren 10000 Patienten getestet und nach ihrer Zulassung engmaschig weiter überprüft. Die Studienprogramme, welche zur Zulassung dieser Medikamente geführt haben, zeigen, wie über die letzten Jahrzehnte Phase III-Studien optimiert wurden, um tatsächlich die Wirkungen, aber vor allem auch die unerwünschten Wirkungen von Medikamenten bereits in der klinischen Prüfung zu erfassen. Es ist beeindruckend zu sehen, wie sich die Daten der Zulassungsstudien nahezu identisch in den Phase IV-Studien reproduzieren lassen. Dabei bestätigt sich, dass die direkten oralen Antikoagulanzien mindestens so effektiv sind wie die Vitamin-K-Antagonisten, jedoch mit einer deutlichen Reduktion schwerer Blutungen. Dies betrifft vor allem die Rate an intrakraniellen Blutungen, die bei den direkten oralen Antikoagulanzien ca. 50\% niedriger ist als bei den Vitamin K-Antagonisten.

Jede gerinnungshemmende Therapie erhöht das Risiko für Blutungen. Besonders dramatisch ist die Situation, wenn eine dringende Operation oder eine Lysetherapie bei Schlaganfall nicht durchgeführt werden können, weil die blutgerinnungshemmende Wirkung des Antikoagulans den Eingriff verbietet. Seit wenigen Monaten steht ein Antidot für Dabigatran zur Verfügung, und in den nächsten 2 Jahren wird voraussichtlich ein Antidot für die Faktor Xa-Inhibitoren zugelassen werden. 
Schritt 4, aktuelle Entwicklungen: Das immer bessere Verständnis der unterschiedlichen Mechanismen, die bei verschiedenen Erkrankungen zu einer verstärkten Blutgerinnung führen, erlaubt es, unterschiedliche Teile der Gerinnungskaskade mit neuen Medikamenten gezielt zu adressieren. Eine effektive Gerinnungshemmung bei gleichzeitig reduziertem Risiko für schwere Blutungen bei Patienten, die auf eine extrakorporale Zirkulation oder auf Unterstützungssysteme wie ein Kunstherz angewiesen sind, sind gegenwärtig Ziel von Forschung und Entwicklung. Die Hemmung von Faktor XII und Faktor XI zeigt bereits beeindruckende Ergebnisse in vorklinischen Experimenten und ersten Phase-I-Studien. Ganz neue Wege werden mit dem Einsatz von „small interfering RNA“ (siRNA) beschritten, die auf der Ebene des Zellkerns die Bildung von Gerinnungsfaktoren gezielt regulieren. Fazit: Störungen der Blutgerinnung, insbesondere thrombembolische Komplikationen, gehören zu den häufigsten Todesursachen und haben einen erheblichen Anteil an der Morbidität einer alternden Bevölkerung. Mit dem demografischen Wandel, der in den nächsten 20 Jahren eine besondere Dynamik aufweisen wird, bekommt eine individuell angepasste Modulation der Blutgerinnungsfähigkeit bei älteren Patienten eine immer größere Bedeutung. Durch 50 Jahre Fortschritt in der Arzneimittel-Therapie zur Prävention und Behandlung von Thrombosen steht Ärzten und Patienten eine Vielzahl von Medikamenten zur Verfügung, die in einer älter werdenden, aber gleichzeitig immer mobileren Bevölkerung eine individuell angepasste Behandlung erlauben. Das kann dem einzelnen Patienten, trotz der Notwendigkeit einer langfristigen Behandlung, große persönliche Freiheiten ermöglichen. Die Hoffnung ist, dass die bessere Verträglichkeit und Wirksamkeit die Akzeptanz einer langfristigen Therapie mit diesen Arzneimitteln erhöht, und damit die Inzidenz von Schlaganfällen und neuen Thrombosen effektiv gesenkt werden kann. Davon profitiert am Ende nicht nur der Patient, sondern das gesamte Gesundheitssystem.

Interessenkonflikte: Berater- oder Gutachtertätigkeit innerhalb der letzten 3 Jahre: Aspen, Boehringer Ingelheim, Merck Sharp and Dome, Bayer Healthcare, Gore Inc.

Honorare, Vortragstätigkeit innerhalb der letzten 3 Jahre: Aspen, Boehringer Ingelheim, MSD, Macopharma, BristolMyers Squibb, Chromatec, Instrumentation Laboratory. Forschungsförderung/Auftragsforschung: Deutsches Rotes Kreuz Blutspendedienst NSTOB; Rovi, Sagent, Biomarin/Prosensa.

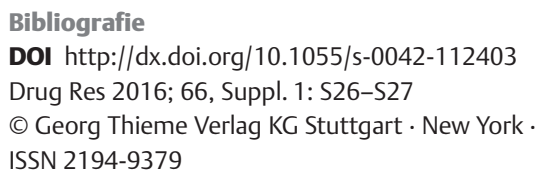

Korrespondenzadresse

Prof. Dr. Andreas Greinacher

Leiter der Abteilung Transfusionsmedizin Institut für Immunologie und

Transfusionsmedizin

Ernst-Moritz-Arndt-Universität Greifswald

Sauerbruchstr.

17487 Greifswald

greinach@uni-greifswald.de

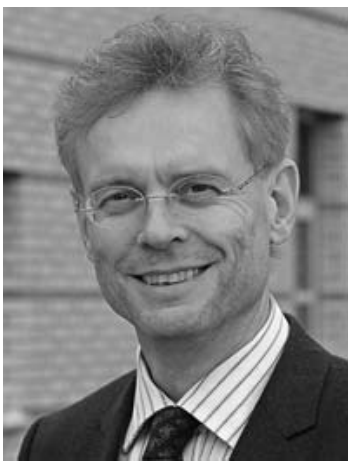

